\title{
Standortkonkurrenz von Lebensmittelmärkten. Frühzeitige Abschätzung und Bewertung der Auswirkungen neuer Märkte
}

\author{
Sascha Anders ${ }^{1}$ \\ Eingegangen: 13. Mai 2017 / Angenommen: 2. Juni 2018 / Online publiziert: 15. Juni 2018 \\ ○) Springer-Verlag GmbH Deutschland, ein Teil von Springer Nature 2018
}

\section{Zusammenfassung}

Die Ansiedlung von Lebensmittelmärkten und deren Wirkungen auf bestehende Nahversorgungsstandorte werden in Deutschland immer wieder kontrovers diskutiert. Bei der Bewertung und Diskussion der möglichen städtebaulichen Auswirkungen neuer Märkte fehlt es aber häufig an objektiven und nachvollziehbaren Bewertungsgrundlagen. Im Rahmen des Forschungsprogramms „Nachhaltiges Landmanagement“ des Bundesministeriums für Bildung und Forschung (BMBF) wurde deshalb ein anwendungsorientiertes, GIS-basiertes Gravitationsmodell entwickelt, das die zu erwartenden Umsatzveränderungen für den Bestand durch die Ansiedlung eines neuen Marktes berechnet. Das Modell ist Grundlage dieses Beitrags. Die Abschätzung der Wirkungen erfolgt mit verhältnismäßig leicht verfügbaren Daten, das Modell ist grundsätzlich deutschlandweit anwendbar. Die Ergebnisse können als zusätzliche Abwägungsvoraussetzungen zu einem frühen Planungszeitpunkt verwendet werden und sollen die Diskussion in den betroffenen Kommunen auf ein solideres Fundament stellen. Im Beitrag werden die Schwierigkeiten bei der Bewertung neuer Lebensmittelmärkte thematisiert, die inhaltlichen und methodischen Hintergründe für das entwickelte Modell erläutert und schließlich eine Einordnung der Ergebnisse vorgenommen.

Schlüsselwörter Einzelhandel · Lebensmittelmarkt · Umsatzumverteilung · Sondergebiet Einzelhandel · Innenbereich · Gravitationsmodell

\section{Competitive Location of Supermarkets. Estimating the Effects of New Markets at an Early Stage}

\begin{abstract}
The locations of supermarkets and their effects on existing local suppliers are a continuous source of controversial discussion. In the debate among experts how to measure the effects and what actually defines urban development deficits, an objective and comprehensible foundation is missing. Therefore as part of the program "Nachhaltiges Landmanagement“ (,Sustainable land management“) of the Bundesministerium für Bildung und Forschung (Federal Ministry of Research and Education (BMBF)) we developed an application-oriented, GIS-based gravity model, which is placed on the anticipated changes in turnover of existing businesses caused by a new supermarket. The appraisal of impacts is achieved with relatively readily available data, the model should, as a rule, work for Germany as a whole. The results can be used as additional assessment at a very early stage, and should give the discussion in the municipalities a better foundation. This paper outlines the difficulties in the judgement of new food markets. It explains the content and methodological background of the developed model, and finally takes a classification of the results.
\end{abstract}

Keywords Retail $\cdot$ Food market $\cdot$ Changes in turnover $\cdot$ Special area retail $\cdot$ Inner area $\cdot$ Gravity model

Sascha Anders

sascha.anders@hcu-hamburg.de

1 Projektentwicklung und Projektmanagement in der Stadtplanung, HafenCity Universität Hamburg, Überseeallee 16, 20457 Hamburg, Deutschland 


\section{Einleitung}

Dieser Beitrag geht auf die Schwierigkeiten ein, die sich bei der planungsrechtlich notwendigen Bewertung und Abwägung neuer Lebensmittelmärkte ergeben. Der Fokus liegt auf der Betrachtung und Diskussion, wie städtebaulich negative Auswirkungen im Sinne des $\S 1$ Abs. $3 \mathrm{BauNVO}^{1}$ und des $\S 34$ Abs. 3 BauGB$^{2}$ auf die vorhandenen Versorgungsstrukturen üblicherweise prognostiziert und bewertet werden. Hierfür werden zunächst kurz die Änderungen auf der Angebots- und Nachfrageseite im Lebensmitteleinzelhandel dargestellt und in einen Zusammenhang zur Entwicklung der baurechtlichen Vorgaben und dem stadtplanerischen Steuerungsanspruch gestellt. Anschließend folgt eine kursorische Betrachtung von Gravitationsmodellen, die im Allgemeinen als Grundlagen für die Berechnung möglicher Verdrängungseffekte auf den Bestand verwendet werden. Dabei wird das gewohnte Verfahren im Genehmigungsprozess, das sich auf die Einholung externer Gutachten konzentriert, kritisch hinterfragt. Thematisiert werden insbesondere die mangelhafte Transparenz der Annahmen und Berechnungen, die normalerweise mit der Komplexität der Berechnungen und der notwendigen individuellen Betrachtung des Marktgeschehens begründet werden. Abschließend wird ein eigenes Bewertungsmodell in seinen Grundzügen vorgestellt und diskutiert. Es wurde als GIS-basiertes Open-source-Werkzeug entwickelt und soll den planenden Kommunen die Möglichkeit geben, eigene Bewertungen von geplanten Lebensmittelmärkten vorzunehmen und die zu erwartenden Umsatzumverteilungen für den Bestand und zu erwartende städtebauliche negative Auswirkungen grob abzuschätzen.

Der Lebensmitteleinzelhandel besitzt als Standort des „Sich-Versorgens“ für die Stadtentwicklung eine große Bedeutung. Lebensmittelmärkte prägen die gewachsenen Stadtteil- und Ortskerne. Ihre im Vergleich zu anderen Branchen hohen Umsatzzahlen (in Deutschland wird knapp die Hälfte aller Ausgaben im Einzelhandel im kurzfristigen Bedarfsbereich umgesetzt; Doplbauer 2015: 5) und die Notwendigkeit, sich diese Güter auch täglich oder wöchentlich beschaffen zu müssen, führen zu hohen Kundenund Passantenfrequenzen. Durch die hohe Kopplungsaffinität der Kunden kann dies zu positiven wirtschaftlichen Effekten für die in der Nähe liegenden Einzelhandelsbetrie-

\footnotetext{
1 Baunutzungsverordnung in der Fassung der Neubekanntmachung vom 21. November 2017.

2 Baugesetzbuch in der Fassung der Neubekanntmachung vom 3. November 2017.
}

be und Dienstleistungseinrichtungen führen. ${ }^{3}$ Neben diesen positiven Agglomerationseffekten sind Supermärkte und Lebensmitteldiscounter auch soziale Orte der Kommunikation, man trifft sich dort und tauscht sich aus (vgl. Krüger/ Anders/Walther et al. 2013: $66 \mathrm{ff}$.).

Für die städtebaulich integrierte Lage von Lebensmittelmärkten sprechen aus stadtplanerischer Sicht noch zwei weitere Punkte: die Sicherstellung der Daseinsgrundvorsorge und die Orientierung der Stadtentwicklung an nachhaltigen und ökologischen Zielen. Unter Sicherstellung der Daseinsgrundvorsorge wird unter anderem eine möglichst wohnortnahe Grundversorgung mit Gütern für den täglichen Bedarf und die Sicherung und Stärkung gewachsener und/oder geplanter städtebaulicher Strukturen, das heißt Innenstädte, Stadtteilzentren und/oder Ortszentren, verstanden (Temmen 2012: 42 ff.). Betrachtet man die ökologischen Effekte, wirken Standorte in städtebaulich integrierter Lage im Vergleich zu Standorten auf der grünen Wiese oder in Gewerbegebieten verkehrsmindernd und führen dazu, dass eher das Fahrrad genutzt oder der Markt zu Fuß aufgesucht wird (,Stadt der kurzen Wege“). Zudem tragen Entwicklungen in gewachsenen Ortslagen dazu bei, der zunehmenden Flächenversiegelung entgegenzuwirken (zur Flächeninanspruchnahme in der Stadtentwicklung vgl. Jörissen/Coenen 2007: $9 \mathrm{ff}$.).

Der Lebensmitteleinzelhandel selbst ist in Deutschland durch eine hohe Dynamik geprägt, die in den vergangenen Jahrzehnten dazu geführt hat, dass sich die Kräfte der räumlichen und betrieblichen Konzentration verstärkt haben. Insgesamt ist der Strukturwandel im deutschen Lebensmitteleinzelhandel mit einem starken Flächenwachstum verbunden, denn neue Märkte wurden häufig an städtebaulich nicht integrierten Standorten entwickelt. In vielen Kommunen und Regionen hat sich auf diese Weise ein sekundäres Versorgungsnetz etabliert, durch das traditionelle Standorte, also gewachsene Zentren, zunehmend unter Druck geraten. Als Konsequenz aus dem Rückgang der absoluten Anzahl der Lebensmittelmärkte und der VergröBerung der Verkaufsfläche an einzelnen Standorten ist das Versorgungsnetz weitmaschiger geworden. Auf der anderen Seite muss man allerdings auch konstatieren, dass mit der Vergrößerung der Ladeneinheiten gleichzeitig auch eine Ausweitung des Angebots einhergeht (vgl. Steinbach 2000:

\footnotetext{
${ }^{3}$ Im Rahmen einer Studie zur qualifizierten Nahversorgung, die diesem Beitrag zugrunde liegt, wurden über 4.000 Haushalte in acht deutschen Kreisen und kreisfreien Städten nach ihrem Einkaufsverhalten befragt. Dabei gaben rund $57 \%$ der Befragten an, dass sie den Einkauf im Lebensmittelmarkt in der Regel mit einer anderen Aktivität koppeln. Neben Einkäufen bei anderen Lebensmittelmärkten werden vor allem Einkäufe bei Bäcker und Metzger, Arztbesuche, Erledigungen bei der Bank, der Post und in der Apotheke genannt (Krüger/Anders/ Walther et al. 2013: 51).
} 
27 ff.; Junker/Kühn 2006: 27 ff.; Kulke 2010: 217 ff.; Reink 2014).

Parallel dazu hat sich in den vergangenen Jahrzehnten das Einkaufsverhalten der Kunden verändert. Es ist wesentlich individueller geworden, und es haben sich Trends wie der Lebensmitteleinkauf mit dem eigenen Pkw (vor allem auf dem Land und in kleinen Städten) und eine Tendenz zum „One-Stop-Shopping“ - also der gesamte Einkauf an einem Ort - durchgesetzt (Kulke 2005: 9 ff.; Junker/Kühn 2006: $42 \mathrm{ff}$.; Merkle 2008: $267 \mathrm{ff}$;; Uttke 2009: $70 \mathrm{ff}$.). Seit einigen Jahren haben allerdings die Wiederentdeckung der (Innen)Stadt als Ort des erlebnisorientierten Konsums und des Städtetourismus, veränderte Arbeitsweisen und Ansprüche an Arbeits- und Wohnorte sowie auch der technische Fortschritt dazu geführt, dass Städte und Innenstädte als Wohn- und Erlebnisräume wieder attraktiver geworden sind. In der Folge wird wieder zunehmend in Zentren investiert. Eine Abkehr von der weiter anhaltenden Flächenentwicklung in den Außenbereichen ist allerdings bislang (noch) nicht zu erkennen (vgl. Brake 2012; Osterhage/Thabe 2012).

\section{Umgang der Stadtplanung mit der Neuansiedlung von Lebensmittelmärkten}

Die kommunale Steuerung bei der Entwicklung und Zulassung von Lebensmittelmärkten erfolgt in Deutschland nach den gesetzlichen Vorgaben des Baugesetzbuches (BauGB) und der Baunutzungsverordnung (BauNVO). Darüber hinaus machen die Bundesländer über ihre Landesentwicklungspläne und/oder Einzelhandelserlasse Vorgaben für die Ansiedlung von Einzelhandelsbetrieben. Teilweise werden diese noch durch Regionalpläne konkretisiert. Die konkrete Festlegung der Zulässigkeit von Einzelhandelsvorhaben obliegt aber der Kommune vor Ort (Planungshoheit der Kommunen). Dabei spielen die gesetzlichen Vorgaben nach $\S 1$ Abs. 3 BauNVO und $\S 34$ Abs. 3 BauGB eine besondere Rolle.

Zur Einordnung der bauplanungsrechtlichen Vorgaben ist ein Blick in die Geschichte hilfreich; dies verdeutlicht den Zusammenhang zwischen dynamischer Flächenentwicklung auf der Anbieterseite und Regelungserfordernis und Regelungsbedarf auf bauplanungsrechtlicher Ebene. So waren bis zur Novellierung der Baunutzungsverordnung 1977 großflächige Einzelhandelsansiedlungen außer in Kerngebieten (MK) auch in Sondergebieten (SO) ohne zusätzliche Prüfung möglich. Mit der Novellierung 1977 wurden zum ersten Mal die Versorgung der Bevölkerung im Einzugsbereich und die Entwicklung zentraler Versorgungsbereiche (Zentren) als schützenswert eingestuft. Auslöser waren die großen SB-Warenhäuser, die in den 1970erJahren in städtebaulich nicht integrierten Lagen entstanden sind und deren (negative) Wirkungen auf die bestehenden Versorgungsstrukturen relativ schnell offensichtlich wurden (Söfker 2013: Rn. 80). Die Grenze zur Großflächigkeit wurde zunächst auf $1.500 \mathrm{qm}$ Geschossfläche festgelegt und im Jahr 1986 mit einer Änderungsverordnung auf 1.200 qm Geschossfläche gesenkt. Ab dieser Grenze wird vermutet (Vermutungsgrenze), dass bestehende Märkte im Einzugsgebiet des neuen Marktes nicht mehr konkurrenzfähig sind und schließen müssen und sich diese Neuansiedlung dann negativ unter anderem auf die Versorgung der Bevölkerung und auf die städtebauliche Struktur bestehender Zentren auswirken kann. Dabei geht die Rechtsprechung davon aus, dass bei einer Geschossfläche von 1.200 qm eine Verkaufsfläche von rund $800 \mathrm{qm}$ erreicht wird (vgl. Krüger/Anders/ Walther et al. 2013: 9). ${ }^{4}$

Großflächige Einzelhandelsbetriebe müssen deshalb unter anderem darlegen, ob von ihrer Ansiedlung positive wie negative Auswirkungen auf die Versorgung der Bevölkerung im Einzugsbereich und die Entwicklung der zentralen Versorgungsbereiche (Zentren) zu erwarten sind (vgl. $\S 1$ Abs. 3 BauNVO). Um die Wirkungen nachweisen oder widerlegen zu können, werden in der Regel Gutachten an private Büros vergeben, teilweise wird auch durch die Genehmigungsbehörde selbst (ohne externes Gutachten) eine begründete Bewertung und Abwägung vorgenommen. Sind negative Auswirkungen im Sinne des § 1 Abs. 3 BauNVO zu erwarten, ist ein großflächiger Einzelhandelsbetrieb nur in einem Kern- (MK) oder Sondergebiet (SO) zulässig. Das bedeutet in der Regel, dass ein neuer Bebauungsplan aufgestellt werden muss. ${ }^{5}$

Bei der Bewertung von Lebensmittelmärkten nach § 1 Abs. 3 BauNVO ist eine differenzierende Betrachtung notwendig. Kuschnerus (2007: 53) betont in diesem Zusammenhang unter anderem die Lage im Stadtkörper und attestiert Standorten in städtebaulich integrierter Lage unter gewissen Voraussetzungen eine städtebauliche Verträglichkeit (vgl. auch BMVBW 2002: 3). Das Bundesverwaltungsgericht hat in seinem Urteil vom 24. November 2005 (4 C 10.04) ausgeführt, dass die Wirkungen der Einzelhandelsbetriebe, die hauptsächlich der wohnortnahen Versorgung dienen, häufig nicht (mehr) mit Hilfe der Großflächigkeit (ab 800 qm Verkaufsfläche) bewertet werden können. Vielmehr sei der Nachweis bzw. die Widerlegung von Auswirkungen im Sinne des $\S 1$ Abs. 3 BauNVO von größerer Bedeutung im Bebauungsplanverfahren und Abwägungsprozess. Weiter hat das Bundesverwaltungsgericht festgelegt, dass hierfür immer eine prognostische Einzelfallbewertung

\footnotetext{
4 Vgl. BVerwG, Urteil vom 22. Mai 1987 - 4 C 19.85; BVerwG, Urteil vom 22. Juli 2004 - 4 B 29.04; BVerwG, Urteil vom 24. November 2005 - 4 C 10.04; vgl. auch BMVBW (2002).

5 BVerwG, Urteil vom 24. November 2005 - 4 C 10.04; vgl. auch BMVBW (2002).
} 
notwendig ist. Dabei ist es grundsätzlich statthaft, die möglichen Auswirkungen anhand von Umsatzumverteilungen und/oder Kaufkraftabflüssen zu beschreiben (vgl. Krüger/ Anders/Walther et al. 2013: 9f.).

Dies vorausgeschickt stellt sich die Frage, ab wann von Auswirkungen im Sinne des $\S 1$ Abs. 3 BauNVO auszugehen ist. Maßgebend in der fachlichen Diskussion darüber sind die Untersuchungen von Vogels, Holl und Birk (1998), die in einer Ex-post-Studie die Auswirkungen von neuen Märkten auf die bestehenden Einzelhandelsstrukturen untersucht haben und unter anderem zu dem Ergebnis kamen, dass ab einer zu erwartenden Umsatzverdrängung von rund $10 \%$ erhebliche städtebauliche Folgen für die Zentren möglich bis wahrscheinlich sind (Vogels/Holl/Birk 1998: 279). Dieser Wert wird seitdem immer wieder kontrovers diskutiert und in der Regel kritisch hinterfragt (vgl. z. B. Acocella/Fürst 2002; Kuschnerus 2007: 168 ff.). Auch die Gerichte haben in verschiedenen Urteilen die notwendige Einzelfallbetrachtung hinsichtlich der relevanten Verträglichkeits- oder Schädlichkeitsschwellen betont und darauf hingewiesen, dass es keine fixe Grenze der Umsatzverdrängung gibt, ab der von Auswirkungen im Sinne des $\S 1$ Abs. 3 BauNVO auszugehen ist und im Streitfall immer das sogenannte Tatsachengericht entscheiden muss. ${ }^{6}$ In der Praxis hat sich aber trotz aller inhaltlichen Kritik eine Beurteilung auf der Grundlage der $10 \%$-Grenze etabliert.

Ähnlich wie in den Regelungen des § 1 Abs. 3 BauNVO, wird auch in $\S 34$ Abs. 3 BauGB argumentiert. Demnach sind Einzelhandelsvorhaben im unbeplanten Innenbereich (also in den Bereichen, in denen es keine Bebauungspläne gibt; sogenannte $§ 34$-Gebiete) nur dann zulässig, wenn durch das Vorhaben keine schädlichen Auswirkungen auf die Zentren (zentralen Versorgungsbereiche) zu erwarten sind. Anders als der $\S 1$ Abs. 3 BauNVO, schützt der $\S 34$ Abs. 3 BauGB die Zentren im Einzugsbereich des Vorhabens gegen sogenannte schädliche Auswirkungen. Ob diese schädlichen Auswirkungen anders zu beurteilen sind als die städtebaulichen Auswirkungen des $\S 1$ Abs. 3 BauNVO, die einen qualifizierten Abstimmungsbedarf im Sinne des $\S 2$ Abs. 2 S. 1 BauGB erfordern, ist in der Fachwelt umstritten (vgl. Jäde/Dirnberger/Weiß 2007: 431). Auch im Fall des $\S 34$ Abs. 3 BauGB ist es vom Einzelfall abhängig, ob schädliche Auswirkungen vorliegen und inwieweit Schädigungen durch bereits vorhandene Märkte in der Beurteilung berücksichtigt werden müssen (Battis/Mitschang/ Reidt 2014: 577). Anders als die Regelungen des $§ 1$ Abs. 3 BauNVO gelten die Vorgaben des $\S 34$ Abs. 3 BauGB jedoch unabhängig von der Größe der Betriebe, das heißt, die Großflächigkeitsgrenze kommt hier nicht zum Tragen (Kuschnerus 2007: 46 ff.; $160 \mathrm{ff} . ; 198$ ).

\footnotetext{
$\overline{6}$ Vgl. BVerwG, Urteil vom 17. Dezember 2009 - 4 C 1.08; BVerwG,
} Urteil vom 11. Oktober 2007 - 45 C 7/07.
Da das Planungsrecht nicht in die wettbewerbliche Auseinandersetzung der einzelnen Betriebe eingreifen darf, argumentiert das Baurecht hier ausschließlich städtebaulich (Kuschnerus 2007: 232 ff.; 262 ff.). Das bedeutet, dass planungsrechtlich relevant Auswirkungen dann sind, wenn sie zu städtebaulichen Missständen führen. Das ist unter anderem der Fall, wenn durch Neuansiedlungen (außerhalb der Zentren) verstärkt Leerstände in den bestehenden Zentren zu erwarten sind, die die vitale städtebauliche Struktur der Zentren erheblich beeinträchtigen. Wie dargelegt, wird in der Fachwelt und auf dem Boden der bisherigen Rechtsprechung immer wieder kontrovers diskutiert, wie diese Wirkungen messbar sind bzw. ab wann von einem städtebaulichen Missstand gesprochen werden kann. Als Beurteilungskriterium hat sich in der Praxis aber der Kaufkraftabzug bzw. der zu erwartende Umsatzverlust für die bestehenden Zentren (und die wohnortnahe Versorgung) durchgesetzt. Das bedeutet auch, dass in der Regel mindestens bei jedem neuen (großflächigen) Lebensmittelmarkt die Vor- und Nachteile detailliert mit den im Planungsprozess zu beteiligenden Akteuren abgewogen werden müssen (Kuschnerus 2007: 168 ff.; Fickert/Fieseler 2008: 842 ff.). ${ }^{7,8}$

\section{Gravitationsmodelle und Verträglichkeitsuntersuchungen}

Auch wenn sogenannte Ex-ante-Gutachten und Prognosen im Rahmen des Bebauungsplanverfahrens durchaus üblich sind (z. B. im Bereich Verkehr oder bei der Bewertung von Umweltauswirkungen), bergen sie bei der Frage nach ökonomischen Auswirkungen eine gewisse Schwierigkeit: Wettbewerbsbeziehungen in der Einzelhandelslandschaft sind äußerst komplex, einfache Ursache-Wirkungs-Zusammenhänge daher eher die Ausnahme. Mit anderen Worten: Um die zu erwartenden städtebaulichen Auswirkungen eines neuen Lebensmittelmarktes abzubilden, ist eine detaillierte Analyse der bestehenden Wettbewerbssituation notwendig. Um diese Auswirkungen zu erheben, werden in der Regel Gutachten an externe Büros vergeben, die bekanntermaßen auf der Grundlage von Gravitations- oder Regressionsmodellen die ökonomische Situation analy-

\footnotetext{
7 Vgl. BVerwG, Urteil vom 11 Oktober 2007 - 4 C 7.07; BVerwG, Urteil vom 17. Dezember 2009 - 4 C 1.08.

8 Relevant sind die planungsrechtlichen Vorgaben grundsätzlich auch für Agglomerationen, das heißt die Ansiedlung mehrerer Lebensmittelmärkte in unmittelbarer Nähe zueinander. Kontrovers diskutiert wird in diesem Zusammenhang der planungsrechtliche Umgang mit mehreren kleinflächigen Einzelhandelsvorhaben nebeneinander, die in der summarischen Betrachtung als großflächiger Einzelhandelsbetrieb angesehen werden können (vgl. Janning 2010). Dies soll hier allerdings nicht weiter thematisiert werden und ist auch nicht Inhalt des entwickelten Modells.
} 
sieren. Darauf aufbauend beschreiben die Gutachten, wie hoch der zu erwartende Umsatz am Vorhabenstandort ist und wie sich dieser „neue“ Umsatz auf die bestehende Situation auswirkt, das heißt, welche Standorte Umsatzverluste zu befürchten haben. Abschließend wird in den Gutachten bewertet, ob durch ein neues Vorhaben städtebauliche Auswirkungen im Sinne des $§ 1$ Abs. 3 BauNVO oder schädliche Auswirkungen im Sinne § 34 Abs. 3 BauGB anzunehmen sind.

Die Gutachten werden in der Regel von der Kommune in Auftrag gegeben, in der ein Vorhaben realisiert werden soll. Es ist durchaus gang und gäbe, dass ein Vorhabenträger ein entsprechendes Gutachten zusammen mit der Bauvoranfrage bei der Kommune vorlegt. In vielen Kommunen gibt es hierzu mittlerweile auch ein festgelegtes Verfahren. Gängig ist zum Beispiel, dass das Gutachten zwar vom Investor bzw. Projektentwickler bezahlt wird, der Gutachter - oder eine Gruppe von möglichen Gutachtern - aber von der Kommune ausgewählt wird.

Auch Gravitationsmodelle sind komplex. Dies kann unter Umständen bedeuten, dass die Annahmen und Berechnungswege, die in den Auswirkungsanalysen zugrunde gelegt werden, in den Gutachten nur unzureichend offengelegt oder Annahmen nicht immer nachvollziehbar begründet werden. ${ }^{9}$ So stellen Müller-Hagedorn, Toporowski und Zielke (2012: $501 \mathrm{ff}$.) bei der Anwendung von Gravitationsmodellen unter anderem heraus, dass die Schätzung des Distanzwiderstandes mit Unsicherheit behaftet sein kann, ebenso die Bewertung der Qualität oder Attraktivität des Angebots. Bei der Auseinandersetzung um die Inhalte von Verträglichkeitsuntersuchungen wird häufig die Vielzahl an Parametern kritisiert, die dazu führen können, dass Annahmen nicht nachvollzogen werden können, das Endergebnis aber maßgeblich beeinflussen. $\mathrm{Zu}$ diesen Parametern gehören unter anderem die räumliche Abgrenzung des Einzugsgebietes und deren Zonierung, die zu erwartenden Umsätze des neuen Vorhabens in Relation zu den Umsätzen der bestehenden Märkte sowie die zusätzliche Berücksichtigung von Potenzialreserven und zukünftigen Kaufkraftzuwächsen. In Streitfällen führt dieser Umstand häufig dazu, dass einem Gutachten (beauftragt beispielsweise von der ansiedelnden Kommune oder dem Investor), das die Unbedenklichkeit des diskutierten Projektes bescheinigt, ein Gegengutachten der benachbarten Kommune mit dazu konträren Ergebnissen folgt. Möglich wird dies, wie dargestellt,

\footnotetext{
9 Dass die Annahmen von den externen Gutachtern nicht vollends offengelegt werden, ist dabei durchaus verständlich. Schließlich würde dadurch detailliertes „Expertenwissen“ preisgegeben.
}

durch unterschiedliche Berechnungsmodelle und durch unterschiedliche Annahmen. ${ }^{10}$

In den Gutachten finden in der Regel unterschiedlich komplexe Gravitationsmodelle ihre Anwendung. Die meisten Modelle zur Modellierung der Kaufkraftströme gehen auf das probabilistische Gravitationsmodell nach Huff (1964) zurück. Mit dem Modell wird die Wahrscheinlichkeit, mit der ein Bewohner eines Wohnortes Einkäufe in einem Markt oder an einem Ort tätigt, ermittelt. Der Ansatz von Huff ermöglicht, Zonen mit unterschiedlicher Kaufwahrscheinlichkeit festzulegen, die über einen Raumwiderstand (das heißt die Entfernung Wohnort-Einkaufsort) abgebildet werden, und jedem Einkaufsort oder Markt eine Attraktivität zuzuweisen. Huff selbst hat dies zum Beispiel über die Verkaufsfläche des jeweiligen Marktes getan. Formal ausgedrückt wird das Gravitationsmodell wie folgt:

$P_{i, j}=\frac{A_{i} \cdot d_{i, j}^{-\lambda}}{\sum_{j=1}^{J} A_{k j} \cdot d_{i, j}^{-\lambda}}$

Dabei drückt $\mathrm{P}_{\mathrm{i}, \mathrm{j}}$ die Wahrscheinlichkeit aus, dass der Kunde am Wohnort $j$ im Markt $i$ einkauft, $d$ bezeichnet die Distanz zwischen den Orten und $A$ die Attraktivität des Marktes. $\lambda$ bildet den Raumwiderstand ab, der individuell angepasst werden kann. Das Huff'sche Modell wurde allerdings ,nicht empirisch abgeleitet, sondern stellt eine theoretische Abstraktion des Kundenverhaltens dar" (Hartmann 2005: 20). Dieses Gravitationsmodell wurde in den folgenden Jahren und Jahrzehnten weiterentwickelt, dabei wurden Möglichkeiten erarbeitet, das Modell durch die Integration weiterer Einflussfaktoren zu modifizieren. Lakshmanan und Hansen (1965) integrierten beispielsweise zur Umsatzschätzung die vorhandene Kaufkraft in die Berechnungen. Nakanishi und Cooper (1974) integrierten eine beliebige Anzahl von Eigenschaften einer Einkaufsstätte wie Ladenimage, Erscheinungsbild und Serviceleistungen in das Gravitationsmodell und entwickelten das „Multiplicative Competitive Interaction Model“ (MCI), das auf Kundenbefragungen und Kundenherkunftsstudien fußt und die Berechnungen aus diesen Attributen einschließt (vgl. Baker 2000: $279 \mathrm{ff}$; Güßefeldt 2002: 351 ff.; Hartmann 2005: 1 ff.; Klein 2007:

\footnotetext{
${ }^{10}$ Die unterschiedlichen Ergebnisse können unter anderem auch durch unterschiedliche Philosophien der Gutachterbüros zustande kommen. So gibt es Unternehmen, die planerischen und steuernden Ansätzen eher kritisch gegenüberstehen und marktliberal argumentieren, und Büros, die sich eher als Unterstützer von planerischen und steuernden, in den Markt eingreifenden Kommunen sehen und neue Ansiedlungen grundsätzlich wesentlich kritischer bewerten. Das spiegelt sich dann auch in den Annahmen, Ergebnissen und den Interpretationen der Ergebnisse wider. Eine Vielzahl an Unternehmen lässt sich aber auch zwischen diesen beiden Polen verorten.
} 
55 ff.). ${ }^{11}$ Kurzum: Auf der Basis des Gravitationsmodells von Huff ist eine Vielzahl von differenzierten Modellen entstanden, die es ermöglichen, sehr viele unterschiedliche Parameter in die Modellberechnungen zu einzubauen. Im Ergebnis machen sie aber die objektive Bewertung des angewandten Berechnungsmodells schwieriger, insbesondere wenn sich zwei Gutachten widersprechen oder zu entgegengesetzten Empfehlungen kommen. Im Zweifel wird es jede Seite verstehen, die eigenen Annahmen und Parameter plausibel darzustellen. Die kommunalen Vertreter - sowohl die kommunalen Planer als auch die politischen Gremien - stehen dann häufig in Anbetracht der Komplexität der durchgeführten Berechnungen vor der Herausforderung, die Plausibilität der einzelnen Annahmen und Berechnungsschritte zu bewerten. Hinzu kommt, dass die beauftragten Gutachter ihren, Werkzeugkasten' (verständlicherweise) nur bis zu einem gewissen Grad offenlegen. Darüber hinaus fehlen den Kommunen Vergleichswerte aus anderen Vorhaben oder zu anderen Standorten, die sie für eine objektive Bewertung der zu erwartenden Auswirkungen hinzuziehen könnten.

Mittlerweile gibt es neben den üblichen beauftragten Verträglichkeitsuntersuchungen - die allerdings häufig nicht öffentlich zugänglich sind - zahlreiche anwendungsorientierte Übertragungen und Weiterentwicklungen der erwähnten Gravitationsmodelle. Schürmann (1999) hat beispielsweise die Auswirkungen eines geplanten SBWarenhauses in Dortmund-Hombruch untersucht und hat neben den beschriebenen Wohnort-Einkaufsort-Relationen zusätzlich Parameter wie Verkaufsflächengröße, Angebotskonzentration im direkten Umfeld und Verkehrsmittelwahl in die Berechnungen aufgenommen. Seine Analyse beinhaltet allerdings mehrere Schätzungen (die nicht empirisch hergeleitet sind) und kann nicht ohne Weiteres auf andere Standorte übertragen werden.

Kanhäußer (2007) setzt sich in seiner Untersuchung sehr detailliert mit den Einzugsbereichen und Wirkungen bestehender Möbelhäuser in zwei deutschen Kleinstädten auseinander und modifiziert auf der Grundlage selbst erhobener empirischer Daten das Huff'sche Gravitationsmodell. Die Ergebnisse belegen zwar eine verbesserte Prognosefähigkeit, es wird aber auch herausgestellt, dass es notwendig ist, zwei zusätzliche Parameter zu kalibrieren. Hierfür sind empirische Daten über Marktanteile und Einkaufswahrscheinlichkeiten notwendig. Eine Übertragbarkeit auf andere Standorte und andere Märkte bzw. die Ableitung von allgemeingültigen Aussagen, ist folglich nicht möglich.
Eine Weiterentwicklung und praktische Anwendung des MCI-Modells von Nakanishi und Cooper (1974) sind auch die Untersuchungen von Wieland (2014). Wieland simuliert unter anderem den Markteintritt von Lebensmittelmärkten in unterschiedlichen Kommunen und modelliert die Auswirkungen auf die bestehenden Kundenströme und Kaufkraftflüsse. Neben den üblichen Parametern Wohn- und Einkaufsort fokussiert Wieland seine Untersuchung vor allem auf Agglomerationseffekte und benutzt als Basis für die Herleitung der Attraktivität und der Einzugsbereiche der einzelnen Märkte eine selbst durchgeführte Haushaltsbefragung. Aufgrund der Komplexität des eigenen Untersuchungsansatzes, der getroffenen Annahmen und untersuchten Hypothesen stellt Wieland (2014: 231) allerdings unter anderem abschließend fest, ,dass über die Hinzunahme zusätzlicher Variablen ein verbesserter Erklärungsgehalt erreicht werden kann [...], jedoch auch ein erhöhtes Risiko von verzerrenden [...] Effekten besteht“. Wieland macht deshalb klar, dass das beste Modell keinesfalls das komplexeste ist (das heißt mit den meisten Erklärungsgrößen) und der Erhöhung der Aussagekraft folglich enge Grenzen gesetzt sind.

Die hier vorgestellte GIS-basierte Modellierung Standortkonkurrenz Supermärkte hat die zuvor skizzierten Modellierungsansätze und Erfahrungen aufgenommen. Handlungsleitend war dabei, die Komplexität der einzugebenden Daten und Einschätzungen zu minimieren und gleichzeitig möglichst belastbare und nachvollziehbare Ergebnisse $\mathrm{zu}$ produzieren. Es erfolgt deshalb eine Konzentration auf die Inhalte des ursprünglichen Huff-Modells, wie es häufig auch heute noch im Planungskontext angewendet wird. Die Variablen wurden auf Distanz und Betriebstyp (in Abhängigkeit von der Verkaufsfläche) eingeschränkt und weitere Einflussgrößen, wie sie beispielsweise im MCI-Modell verwendet werden, bewusst außen vor gelassen. Mit diesem Modell soll versucht werden, die Komplexität der Berechnungen zu minimieren und den Einfluss möglicher Verzerrungen durch zusätzliche Parameter auszuschließen. Das soll die Anwendbarkeit (auch durch die Reduktion von benötigten Daten) verbessern. Das Gravitationsmodell erzeugt auf der Grundlage eigener empirischer Untersuchungen zum Einkaufsverhalten und auf einer Basis von relativ leicht zugänglichen Datensätzen Prognosen, die mögliche Umsatzumverteilungen und städtebauliche Auswirkungen durch neue Lebensmittelmärkte aufzeigen. Der Anspruch des entwickelten Tools ist, die Berechnungen weitestgehend automatisiert durchzuführen, die Annahmen offenzulegen und deutschlandweit anwendbar zu sein.

${ }^{11}$ Darüber hinaus gibt es zahlreiche weitere Untersuchungen und Publikationen, die sich mit der Anwendung und Weiterentwicklung von Gravitationsmodellen beschäftigen, z. B. Li/Liu (2012) oder De Beule/Van den Poel/Van de Weghe (2014). Hierauf wird im Folgenden aber nicht weiter eingegangen. 


\section{Das Modell „Standortkonkurrenz Supermärkte"}

Im Fokus des Modells Standortkonkurrenz Supermärkte stehen die zu erwartenden Veränderungen der Kaufkraftbindung - und somit die zu erwartenden Umsatzveränderungen für den Bestand -, die durch die Ansiedlung eines neuen Lebensmittelmarktes zu erwarten sind.

Der Grundgedanke ist dabei, den Entscheidungsträgern vor Ort in einer sehr frühen Phase deutlich vor Beginn des Bebauungsplanverfahrens eine erste überschlägig ermittelte, objektive Abwägungsgrundlage an die Hand zu geben. Anders als bei den ,üblichen“ Verträglichkeitsuntersuchungen trifft das hier vorgestellte Berechnungsmodell keine Entscheidung für oder gegen einen Standort oder ein Vorhaben und nimmt den zuständigen oder betroffenen Akteuren vor Ort keine Planungs- oder Genehmigungsentscheidungen ab. Das GIS-basierte Modell ermöglicht es, die Standorte, Verkaufsflächen und Anbieter zu in der Diskussion stehenden Planungen zu variieren, auf ihre unterschiedlichen Auswirkungen zu untersuchen und deren Wirkungen miteinander zu vergleichen. Später erforderliche, auf den spezifischen Einzelfall bezogene Fachgutachten (zu den städtebaulichen Auswirkungen) kann das Berechnungstool nicht ersetzen. Sie können aber anhand der Voruntersuchung auf Plausibilität geprüft werden.

Die Modellberechnungen basieren auf den Ergebnissen einer telefonischen Haushaltsumfrage, die im Rahmen einer vorausgegangenen Untersuchung zur qualifizierten Nahversorgung durchgeführt wurde (zur genaueren Erläuterung des Settings vgl. Krüger/Anders/Walther et al. 2013: $17 \mathrm{ff}$.). Im Rahmen der telefonischen Haushaltsbefragung wurden in unterschiedlichen siedlungsstrukturellen Kontexten über 4.000 Haushalte (rund 10.500 Personen) zu insgesamt über 6.100 Einkaufsvorgängen (Angabe des ersten und zweiten Haupteinkaufsortes) befragt. Die georeferenzierte Erfassung der Nachfrage- und Angebotssituation vor Ort (das heißt der Wohnorte der befragten Personen, der aufgesuchten Lebensmittelmärkte und des Bestandes der Lebensmittelmärkte vor Ort) ermöglichte es, die Konkurrenzsituation vor Ort abzubilden und das Einkaufsverhalten genau zu untersuchen. Neben der Frage nach dem Einkaufsort wurden unter anderem auch die Einkaufshäufigkeit, die durchschnittlichen Ausgaben je Einkauf und die HaushaltsgröBe abgefragt. Auf dieser Grundlage konnte näherungsweise abgebildet werden, unter welchen Bedingungen (StadtgröBe, Konkurrenzsituation) welcher Markt (differenziert nach Anbieter und Größe) aufgesucht wird und wie hoch die jeweiligen Ausgabenanteile sind.

Darauf aufbauend wurden mit der Statistik- und Analyse-Software SPSS differenzierte Auswertungen durchgeführt, in denen neben den unterschiedlichen Betriebstypen auch die spezifische Konkurrenzsituation abgebildet wurde. Die Auswertungen erfolgten nach dem folgenden Muster:

- Anteil der befragten Personen, die einen Markt des Anbieters $x y$ aufsuchen, wenn der nächste Markt des Anbieters $x y$ maximal 1.000/2.000 m (usw.) von der Wohnung entfernt liegt.

- Anteil der befragten Personen, die einen Markt des Anbieters $x y$ aufsuchen, wenn der nächste Markt des Anbieters $x y$ maximal 1.000/2.000 m (usw.) von der Wohnung entfernt liegt und kein weiterer Markt eines anderen Anbieters vorhanden ist.

Daraus konnte in Abhängigkeit vom Betriebstyp (differenziert nach Anbieter und Größe des Marktes) und der Entfernung zwischen Wohnort und Lebensmittelmarkt näherungsweise die Einkaufswahrscheinlichkeit eines Haushalts/einer Person zum jeweiligen Markt abgeleitet werden. In der Summe der Auswertungen wurden diese näherungsweise als Exponentialfunktion abgebildet. ${ }^{12}$

Abbildung 1 stellt die durchschnittliche Kaufkraftbindung für ausgewählte Betriebstypen in Abhängigkeit von der Entfernung Wohnort-Markt für Standorte in Städten und Gemeinden zwischen 20.000 und 50.000 Einwohnern dar. ${ }^{13}$

In die Berechnungen wurde zusätzlich ein Attraktivitätsfaktor integriert, der die Konkurrenz zweier Märkte des gleichen Anbieters berücksichtigt (Kannibalisierungseffekte). Um die Komplexität der Berechnungen und die notwendigen Eingaben möglichst gering zu halten, wurde darüber hinaus auf die Einbeziehung weiterer Faktoren wie positive Kopplungs- und Agglomerationseffekte verzichtet. Hier wären genauere Analysen notwendig gewesen, zum Beispiel ab welcher Entfernung zueinander positive Effekte zu verzeichnen sind und wie sich andere Infrastrukturen (Ärzte, Schulen, Behörden, andere Einkäufe, Arbeitsort etc.) auf die Wahl des Einkaufsortes auswirken. ${ }^{14}$ Es ist

\footnotetext{
12 Es gibt in der Literatur zahlreiche Untersuchungen und Diskussionen darüber, welche mathematischen Funktionsformen die reale Abnahme der Marktanteile mit der Entfernung am besten abbilden. Dabei werden neben der Exponentialfunktion zum Beispiel auch die Potenzfunktion oder die Arcustangensfunktion diskutiert. In diesem Beitrag wurde die Exponentialfunktion verwendet, weil sie aus Sicht des Autors die reale Situation am besten näherungsweise abbilden kann. Hierüber kann man sicher streiten, das soll aber nicht der Schwerpunkt dieses Beitrags sein (vgl. Kanhäußer 2007).

${ }^{13}$ Für die Ableitung betriebstypenspezifischer Kaufkraftbindung werden im Modell die folgenden Betriebstypen differenziert: Aldi, Lidl, Penny, Norma, sonstige Discounter, kleine Supermärkte (Edeka/Rewe) bis $800 \mathrm{qm}$, mittelgroße Supermärkte (Edeka/Rewe) 801 bis $1.500 \mathrm{qm}$, große Supermärkte (Edeka/Rewe) 1.501 bis 2.500 qm Verkaufsfläche, Verbrauchermärkte 2.501 bis 5.000 qm Verkaufsfläche und SB-Warenhäuser ab 5.001 qm Verkaufsfläche, kleiner Nahversorger unter 400 qm Verkaufsfläche. Außerdem erfolgt eine Differenzierung nach Stadtgröße.
}

${ }^{14} \mathrm{Zu}$ den Wirkungen von Agglomerationen vgl. z. B. Wieland (2014). 
Abbildung 1 Kaufkraftbindung nach ausgewählten Betriebstynung Wohnort-Markt, Städte ab 20.001 bis 50.000 Einwohner pen in Abhängigkeit der Entfer-

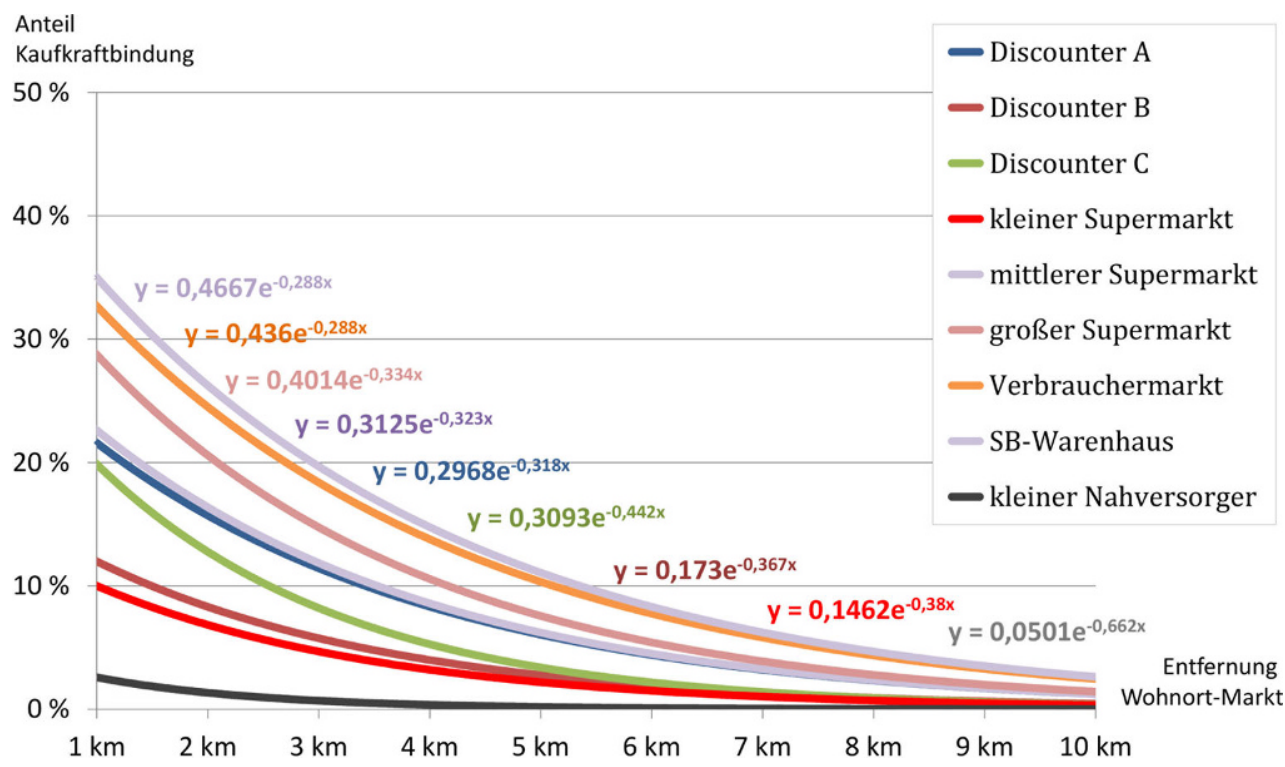

aber im Modell grundsätzlich möglich, die zu erwartenden Einzugsbereiche von neuen Lebensmittelmärkten, die als Koppelstandort entwickelt werden, abzubilden und deren Wirkungen zu prognostizieren.

Das GIS-basierte Modell setzt diese kurz skizzierten Annahmen zur Einkaufswahrscheinlichkeit in Bezug zur Einwohnerzahl und zur Kaufkraft vor Ort und errechnet daraus den wahrscheinlichen Umsatz pro Lebensmittelmarkt. Basis hierfür ist ein Raster von Siedlungszellen mit einer Kantenlänge von 250 mal 250 Metern. Die Konkurrenzsituation wird dabei durch die Lage der Märkte zueinander und die Distanz zu den Wohnorten (Siedlungszellen) der Kunden abgebildet. Grundlage ist das örtliche Straßennetz, das Routing Markt-Siedlungszelle erfolgt während der Anwendung.

Die Berechnungsschritte des beschriebenen Gravitationsmodells können mit der folgenden Formel abgebildet werden:

$U_{j}=\sum_{i=1}^{I} C_{i} \frac{A_{j} \cdot d_{i j}^{-\lambda}}{\sum_{j=1}^{J} A_{j} \cdot d_{i j}^{-\lambda}}$

Dabei bezeichnet $U_{j}$ den Umsatz des Lebensmittelmarktes am Standort $j$. $C_{i}$ bildet die vorhandene Kaufkraft am Wohnort $i$ ab. $A$ bezeichnet die Attraktivität und $\lambda$ den Distanzexponenten, also die Einkaufswahrscheinlichkeit am Standort, die in unserem Modell nach Betriebstypen differenziert wird. Die Distanz zwischen Wohnort (Siedlungszelle) und Lebensmittelmarkt wird durch $d_{i, j}$ dargestellt.

Innerhalb des Modells werden drei übergeordnete Rechenschritte durchgeführt:
- Schritt A: Berechnung des wahrscheinlichen Umsatzes pro Standort (Lebensmittelmarkt) innerhalb des Betrachtungszeitraums (Ist-Situation)

- Schritt B: Berechnung des wahrscheinlichen Umsatzes pro Standort (Lebensmittelmarkt) innerhalb des Betrachtungsraums einschließlich Berechnung des zu erwartenden Umsatzes eines neuen, geplanten Lebensmittelmarktes am Standort $x y$. Hierdurch ergeben sich im Vergleich zu Schritt A durch die neue Konkurrenzsituation niedrigere Umsätze für die Bestandsmärkte.

- Schritt C: Delta-Betrachtung: Berechnung der Differenz der Kaufkraftbindung und der zu erwartenden Umsätze vor und nach Realisierung des neuen Marktes (Differenz aus Schritt A und B).

Wie ausgeführt, kann planerisch und rechtlich nur sehr schwierig begründet werden, ab wie viel Prozent Umsatzumverteilung von städtebaulichen Auswirkungen im Sinne des $\S 1$ Abs. 3 BauNVO oder $\S 34$ Abs. 3 BauGB auszugehen ist. Zur Bewertung werden deshalb zusätzlich die Ausstattungskennziffern „Verkaufsfläche pro Einwohner“ und „Einzelhandelszentralität“ (Umsatz-KaufkraftRelation) $)^{15}$ ausgegeben.

Im Modell werden grundsätzlich alle Lebensmittelmärkte innerhalb der Betrachtungsregion berücksichtigt. Es ist möglich, die Wirkungen auf einzelne Zentren, also Innen-

\footnotetext{
15 Unter Einzelhandelszentralität wird das Verhältnis der vor Ort erwirtschafteten Einzelhandelsumsätze zum vorhandenen Kaufkraftpotenzial einer Kommune (oder innerhalb einer Region) beschrieben. Werte über $100 \%$ drücken aus, dass die Kommune/die Betrachtungsregion saldierte Umsatzzuflüsse aufweist, Werte unter $100 \%$ weisen auf saldierte Kaufkraftabflüsse hin. Zur Definition von Einzelhandelszentralität vgl. gif 2014: $191 \mathrm{f}$.
} 


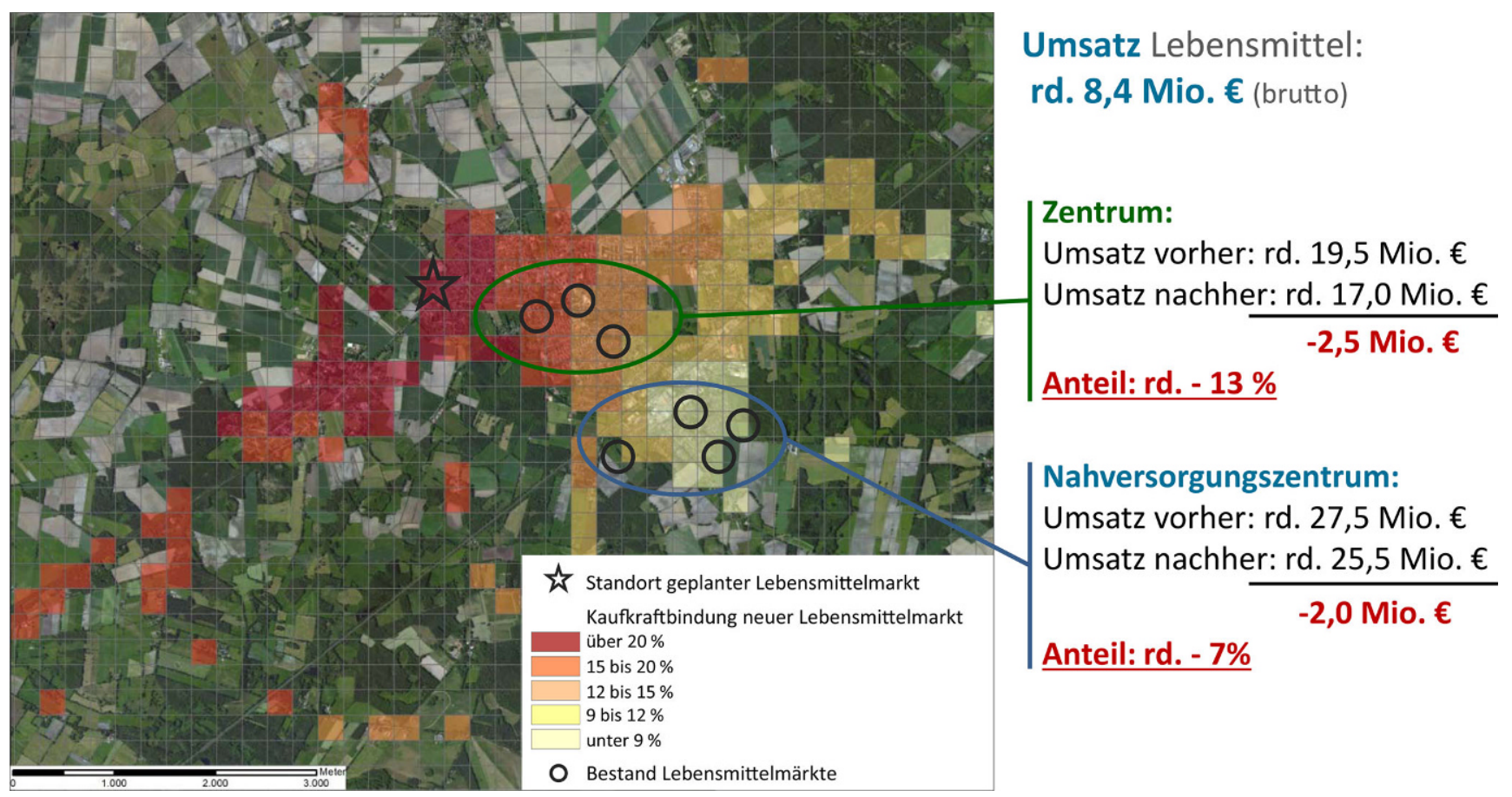

Abbildung 2 Kaufkraftbindung des neu geplanten Marktes auf Basis der Kaufkraft und der Einwohner pro Siedlungszelle (250 m x $250 \mathrm{~m}$ ) sowie Einkaufswahrscheinlichkeit zum geplanten Vorhaben und zu den bestehenden Lebensmittelmärkten. Quelle: Eigene Darstellung, Kartengrundlage: ESRI Grundkarte

städte, Stadtteil- und Nahversorgungszentren sowie einzelne bedeutende Versorgungsstandorte herauszustellen und gesondert zu betrachten.

Eine Beispielrechnung für den Ansiedlungsfall eines Lebensmittelmarktes ergibt das folgende Einzugsgebiet differenziert nach anteiliger Kaufkraftbindung (vgl. Abbildung 2). Für den neuen Lebensmittelmarkt kann in diesem Beispiel ein zu erwartender jährlicher Gesamtumsatz von rund 8,4 Mio. Euro im Lebensmittelbereich ermittelt werden. ${ }^{16}$ Aus der Delta-Betrachtung vor und nach der Realisierung des Planvorhabens errechnet sich eine Umsatzumverteilung von rund $13 \%$ im angrenzenden Zentrum und rund $7 \%$ im südlich gelegenen Nahversorgungszentrum.

Zur Bewertung der zu erwartenden Umsatzveränderungen im Bestand liefert das Modell für diesen Beispielfall zusätzlich die folgenden Ausstattungskennziffern, die den Abwägungsprozess vor Ort unterstützen können: Anstieg der Verkaufsflächenausstattung im Lebensmittelbereich von 0,35 auf 0,38 qm pro Einwohner und Anstieg der Einzelhandelszentralität im Lebensmittelbereich von $87 \%$ auf $90 \%$.

Zur Vergleichbarkeit und Bewertung innerhalb der Region werden zusätzlich die folgenden Vergleichszahlen für die angrenzenden Kommunen errechnet. Hieraus kann ab-

\footnotetext{
${ }^{16}$ Die zu erwartenden Umsätze für den Bereich Drogeriewaren sowie im Nonfood-II-Bereich (also Aktionsware wie Textilien) sind in den Berechnungen nicht enthalten.
}

geleitet werden, ob der Ausstattungsgrad der betroffenen Kommune im regionalen Vergleich bislang eher über- oder unterdurchschnittlich ist (vgl. Abbildung 3).

Um die beschriebenen Berechnungen durchführen zu können, ist neben der Eingabe relevanter Projektdaten für das neue Vorhaben (Standort, Anbieter, Verkaufsflächengröße), lediglich das Einpflegen einer Bestandsdatenbank der bestehenden Lebensmittelmärkte in der Region notwendig. Alle weiteren Berechnungen (Ermittlung der Einwohnerzahlen im Einzugsgebiet, Ermittlung der vorhandenen Kaufkraft, Routing Siedlungszelle-Lebensmittelmarkt und die Umsatzberechnungen auf der Grundlage des Gravitationsmodells) erfolgen dann automatisiert im System. Es bleibt aber grundsätzlich möglich, sämtliche voreingestellte Annahmen, z. B. zum Distanzwiderstand oder zur Attraktivität der Märkte, individuell anzupassen.

\section{Bewertung und Weiterentwicklung des Modells "Standortkonkurrenz Supermärkte"}

Das Modell Standortkonkurrenz Supermärkte wurde im Rahmen des Forschungsprogramms „Nachhaltiges Landmanagement" des Bundesministeriums für Bildung und Forschung (BMBF) im Zeitraum 2011 bis 2015 gefördert und ist ein Verbundprojekt im Modul B „Innovative Sys- 
Abbildung 3 Kennziffern: Kaufkraft (brutto), Umsatz (brutto), Zentralität Lebensmittel, Einwohner, Verkaufsfläche im Bereich Lebensmittel und Verkaufsfläche pro Einwohner innerhalb einer Beispielregion

\begin{tabular}{|c|c|c|c|c|}
\hline Kommunen & $\begin{array}{c}\text { Kaufkraft } \\
\text { (brutto) }\end{array}$ & $\begin{array}{l}\text { Umsatz } \\
\text { (brutto) }\end{array}$ & $\begin{array}{c}\text { Zentralität } \\
\text { Lebensmittel }\end{array}$ & $\begin{array}{l}\quad \text { qm } \\
\text { Verkaufsfläche } \\
\text { pro Einwohner }\end{array}$ \\
\hline Kommune $\mathrm{A}$ & $89.171 .642 €$ & $106.128 .885 €$ & $119,0 \%$ & 0,50 \\
\hline Kommune B & $27.186 .309 €$ & $18.973 .706 €$ & $69,8 \%$ & 0,38 \\
\hline Kommune C & $30.856 .929 €$ & $17.307 .115 €$ & $56,1 \%$ & 0,26 \\
\hline Kommune D & $24.911 .848 €$ & $20.978 .075 €$ & $84,2 \%$ & 0,30 \\
\hline Kommune E & $24.512 .620 €$ & $23.994 .422 €$ & $97,9 \%$ & 0,28 \\
\hline$\ldots$ & $\ldots$ & $\ldots$ & $\ldots$ & $\ldots$ \\
\hline $\begin{array}{l}\text { Betrachtungsraum, } \\
\text { Landkreis }\end{array}$ & $565.738 .496 €$ & $540.272 .943 €$ & $95,5 \%$ & 0,42 \\
\hline
\end{tabular}

temlösungen für ein nachhaltiges Landmanagement ". ${ }^{17}$ Das vorgestellte Modell ist Teil des Forschungsvorhabens „RegioProjektCheck“. RegioProjektCheck wurde von einem Forschungsverbund, bestehend aus der HafenCity Universität Hamburg (HCU) und dem Institut für Landes- und Stadtentwicklungsforschung (ILS), Dortmund, in Kooperation mit dem Planungsbüro Gertz Gutsche Rümenapp (GGR), bearbeitet und wird seit Ende 2016 unter dem neuen Namen „ProjektCheck“ fortgeschrieben und weiterentwickelt. Neben der Beseitigung einiger technischer Fehler, die sich bei der Anwendung gezeigt haben, wird versucht, das Modell Standortkonkurrenz Supermärkte anwendungsfreundlicher zu gestalten. ${ }^{18}$

Vergleicht man den hier vorgestellten Ansatz mit den bislang vorliegenden Analysen und Weiterentwicklungen von Gravitationsmodellen und deren Anwendung, zeichnet sich das Modell durch die Integration in ein GIS-Modell, die freie Verfügbarkeit und die damit zusammenhängende Möglichkeit aus, grundsätzlich bundesweit anwendbar zu sein. Außerdem basieren die Annahmen in der Gravitation auf einer sehr breit angelegten empirischen $\mathrm{Ba}$ sis, die eine gewisse Belastbarkeit der Ergebnisse erwarten lässt. Für den Anwender bedeutet dies, dass die individuelle Abschätzung des Einzugsgebietes, des Distanzwiderstandes oder die Bewertung der Attraktivität eines neuen Marktes nicht notwendig ist, da dies automatisiert durch das Modell abgebildet wird. Das bedeutet allerdings auch, dass das Modell nicht auf spezifische Qualitäten an besonderen Orten eingeht. Obwohl es grundsätzlich möglich ist, die Parameter durch die Offenheit des Modells zu verändern, war dies allerdings auch die Intention des Ansatzes. Denn damit soll ausgeschlossen werden, je nach Interessenlage oder subjektiver Einschätzung, einzelne Parameter so zu kalibrieren, um das gewünschte Ergebnis zu erhalten. Das

17 Vgl. http://www.nachhaltiges-landmanagement.de (22.04.2018).

18 Für mehr Informationen zum ProjektCheck und zur Anwendung vgl. http://www.projekt-check.de (22.04.2018) und die Abschlussbroschüre zu RegioProjektCheck (vgl. HCU/ILS 2014). Die GIS-Anwendung ist außerdem als Open-Source-Datei frei auf der Projekt-Homepage verfügbar.
Modell wurde bislang in mehreren Kommunen angewendet und die Ergebnisse waren bisher ausnahmslos plausibel. $\mathrm{Ob}$ das für alle Regionen und siedlungsstrukturellen Kontexte gilt, kann nicht mit Bestimmtheit gesagt werden. Es wird aber angestrebt, das Tool in nächster Zeit weiter zu testen und dadurch belastbarer zu machen.

Durch die automatisierte Anwendung des hinterlegten Gravitationsmodells ist zwar keine Abgrenzung des Einzugsgebietes notwendig, allerdings muss bei der Auswahl der Betrachtungsregion sichergestellt werden, dass diese deutlich größer als die Einzugsbereiche der einzelnen Märkte ist. Für den Fall, dass die relevanten Konkurrenzmärkte in der Nachbarkommune nicht in das Modell eingespeist werden, wird die Bestands- und Versorgungssituation der Kommune $\mathrm{zu}$ positiv dargestellt (da die interkommunale Konkurrenzsituation ausgeblendet wird). Wenn die Bevölkerungsverteilung der angrenzenden Kommunen nicht in das Modell integriert wird, führt das in der Tendenz zu einer Unterbewertung der Leistungskennziffern der eigenen Lebensmittelmärkte (da das Kaufkraftpotenzial außerhalb der eigenen kommunalen Grenzen nicht in die Berechnungen einfließen kann). Aus diesem Grund liefert das Modell am Rand des Betrachtungsraums immer ungenaue Ergebnisse. Es sollte bei der Anwendung des Modells deshalb immer sichergestellt werden, dass die regionale Konkurrenzsituation durch die Eingabe einer regionalen Bestandsdatenbank abgebildet wird.

Die methodische Diskussion um die Modellierung von Kaufkraftströmen war in der Vergangenheit vor allem darauf ausgerichtet, die Einkaufsbeziehungen möglichst genau abbilden zu können. Auf Basis des Huffschen Gravitationsmodells hat es in den vergangenen Jahrzehnten eine Vielzahl von Ansätzen gegeben, möglichst viele Parameter in die Berechnungen zu integrieren. Die jeweiligen Modelle wurden dadurch zwar (im theoretisch-wissenschaftlichen Sinn) genauer, häufig fehlte es aber an der empirischen Basis bzw. an dem Knowhow, die einzelnen Parameter auf Grundlage einer fundierten empirischen Basis genau zu kalibrieren. Zudem haben einige Modelle den Anspruch, das Einkaufsverhalten generell (das heißt unabhängig von der 
jeweiligen Branche) abbilden zu wollen. Hier wurde sich dem Thema Modellierung von Kaufkraftströmen quasi von der anderen Seite genähert und ein anwendungsbezogenes, empiriegeleitetes und auf wenigen Indikatoren beruhendes Modell entwickelt, das sich zum einen auf eine Branche konzentriert (Lebensmittel), und zum anderen nicht den Anspruch hat, jede spezifische Situation mit Agglomerationsvorteilen, Kopplungen mit anderen Nutzungen und spezifischen Einkaufsgewohnheiten genau beurteilen zu können (das dürfte im Übrigen auch mit dem kompliziertesten Modell schwierig werden; vgl. Wieland 2014: 231). Das vorliegende Modell gibt dem Anwender aber die Möglichkeit, mit relativ wenig Aufwand eine mögliche Planungssituation frühzeitig abzuschätzen. Dabei profitiert es auch von seiner technischen Leistungsfähigkeit, die Kaufkraftbindung je Siedlungszelle mit den Lebensmittelmärkten in einer Region zu verschneiden. Daraus können (im Grunde deutschlandweit) spezifische Konkurrenzsituationen abgebildet und Kennziffern abgeleitet werden. Auf die recht komplizierten Vorgaben aus Baugesetzbuch und Baunutzungsverordnung und die nicht immer eindeutigen Rechtsprechungen hierzu liefert das Modell zwar keine Antworten, es kann aber zu einer Verbesserung der Planungs- und Entscheidungsverfahren auf kommunaler und regionaler Ebene beitragen und eine nachhaltige - weil flächen- und infrastrukturschonende - Planung unterstützen.

Die bisherigen Erfahrungen und Rückmeldungen zu den Ergebnissen zeigen, dass Kommunen das Modell vor allem intern zur Beratung nutzen (würden), um Standorte und Vorhabenvarianten miteinander zu vergleichen. Kreise und andere regionale Gebietskörperschaften achten bei der Bewertung von neuen Vorhaben vor allem auf die Ausgewogenheit der Entwicklung innerhalb der Gemeinden und im regionalen Kontext - immer mit dem Fokus, die bestehenden Versorgungszentren zu sichern und zu stärken. $\mathrm{Ob}$ das Modell in Zukunft von den Kommunen und Verbänden genutzt wird, wird sich zeigen und neben der Plausibilität der erzielten Ergebnisse davon abhängig sein, ob es gelingt, die Anwendung des Modells Standortkonkurrenz Supermärkte möglichst nutzerfreundlich und leicht verständlich zu gestalten.

\section{Literatur}

Acocella, D.; Fürst, U. C. (2002): 10 Prozent Umsatzumverteilung. Ein einheitlicher Bewertungsmaßstab für die städtebauliche und raumordnerische Verträglichkeit großflächiger Einzelhandelsbetriebe? In: BAG-Handelsmagazin 5/6, 29-31.

Baker, R. G. V. (2000): What Underpins the Gravity Coefficient in Space-Time Modelling Aggregate Consumer Trip Behaviour to Shopping Centres? In: Reggiani, A. (Hrsg.): Spatial Economic Science. New Frontiers in Theory and Methodology. Berlin, 279302.

Battis, U.; Mitschang, S.; Reidt, O. (2014): Baugesetzbuch Kommentar. München.
BMVBW - Bundesministerium für Verkehr, Bau- und Wohnungswesen (2002): Bericht der Arbeitsgruppe Strukturwandel im Lebensmitteleinzelhandel und $\S 1$ Abs. 3 BauNVO. Berlin.

Brake, K. (2012): Berlin - relative Reurbanisierung einer gewendeten Stadt. In: Brake, K.; Herfert, G. (Hrsg.): Reurbanisierung. Materialität und Diskurs in Deutschland. Wiesbaden, 258-286. doi: 10.1007/978-3-531-94211-7

De Beule, M.; van den Poel, D.; van de Weghe, N. (2014): An extended Huff-model for robustly benchmarking and predicting retail network performance. In: Applied Geography 46, 1, 80-89. doi: 10.1016/j.apgeog.2013.09.026

Doplbauer, G. (2015): Ecommerce. Wachstum ohne Grenzen? OnlineAnteile der Sortimente - heute und morgen. Bruchsal.

Fickert, H. C.; Fieseler, H. (2008): Baunutzungsverordnung. Kommentar unter besonderer Berücksichtigung des deutschen und gemeinschaftlichen Umweltschutzes mit ergänzenden Rechts- und Verwaltungsvorschriften. Stuttgart.

gif - Gesellschaft für immobilienwirtschaftliche Forschung (2014): Definitionen zur Einzelhandelsanalyse. Definitionen für die Beurteilung von Einzelhandelsimmobilien und Geschäftslagen sowie für die Erstellung von Einzelhandelsanalysen, Auswirkungsgutachten und kommunalen Einzelhandelsgutachten. Wiesbaden.

Güßefeldt, J. (2002): Zur Modellierung von räumlichen Kaufkraftströmen in unvollkommenen Märkten. In: Erdkunde 56, 4, 351-370. doi: 10.3112/erdkunde.2002.04.02

Hartmann, M. (2005): Gravitationsmodelle als Verfahren der Standortanalyse im Einzelhandel. Halle. $=$ Statistik Regional Electronic Papers SR-2005-02.

HCU - HafenCity Universität Hamburg; ILS - Institut für Landes- und Stadtentwicklungsforschung (2014): RegioProjektCheck. Werkzeuge zur regionalen Evaluierung geplanter Projekte für Wohnen, Gewerbe und Einzelhandel im Rahmen eines nachhaltigen Landmanagements. Abschlussbroschüre November 2014. Hamburg/Dortmund.

Huff, D. L. (1964): Defining and Estimating a Trading Area. In: Journal of Marketing 28, 3, 34-38. doi: 10.2307/1249154

Jäde, H.; Dirnberger, F.; Weiß, J. (2007.): Kommentar BauGB, BauNVO. Stuttgart.

Janning, H. (2010): Regelungen zur Art der baulichen Nutzung - Einzelhandel und zentrale Versorgungsbereiche. In: BMVBS - Bundesministerium für Verkehr, Bau und Stadtentwicklung (Hrsg.): Berliner Gespräche zum Städtebaurecht. Band II: Dokumentation Festveranstaltung/Materialien. Berlin, 113-124.

Jörissen, J.; Coenen, R. (2007): Sparsame und schonende Flächennutzung. Entwicklung und Steuerbarkeit des Flächenverbrauchs. Berlin.

Junker, R.; Kühn, G. (2006): Nahversorgung in Großstädten. Berlin. Difu-Beiträge zur Stadtforschung 47.

Kanhäußer, C. (2007): Modellierung und Prognose von Marktgebieten am Beispiel des Möbeleinzelhandels. In: Klein, R.; Rauh, J. (Hrsg.): Analysemethodik und Modellierung in der geographischen Handelsforschung. Passau, 75-110. = Geographische Handelsforschung 13.

Klein, R. (2007): Der probabilistische Ansatz zur Modellierung des räumlichen Einkaufsverhaltens. In: Klein, R.; Rauh, J. (Hrsg.): Analysemethodik und Modellierung in der geographischen Handelsforschung. Passau, 55-74. = Geographische Handelsforschung 13.

Krüger, T.; Anders, S.; Walther, M.; Klein, K.; Segerer, M.; (2013): Qualifizierte Nahversorgung im Lebensmitteleinzelhandel. Endbericht. Hamburg/Regensburg.

Kulke, E. (2005): Räumliche Konsumentenverhaltensweisen. In: Kulke, E. (Hrsg.): Dem Konsumenten auf der Spur. Neue Angebotsstrategien und Nachfragemuster. Passau, 9-25. = Geographische Handelsforschung 11.

Kulke, E. (2010): Strukturwandel im Einzelhandel. In: Kulke, E. (Hrsg.): Wirtschaftsgeographie Deutschlands. Heidelberg, 217232. 
Kuschnerus, U. (2007): Der standortgerechte Einzelhandel. Bonn.

Lakshmanan, J. R.; Hansen, W. G. (1965): A Retail Market Potential Model. In: Journal of the American Institute of Planners 31, 2, 134-143. doi: 10.1080/01944366508978155

Li, Y.; Liu, L. (2012): Assessing the impact of retail location on store performance: A comparison of Wal-Mart and Kmart stores in Cincinnati. In: Applied Geography 32, 2, 591-600. doi: 10.1016/j.apgeog.2011.07.006

Merkle, W. (2008): Der Mythos vom „Tod der Mitte“ - Handlungsfelder für eine weiterhin erfolgreiche Marktbearbeitung. In: Kreutzer, R. T.; Merkle, W. (Hrsg.): Die neue Macht des Marketing. Wie Sie Ihr Unternehmen mit Emotion, Innovation und Präzision profilieren. Wiesbaden, 267-290.

Müller-Hagedorn, L.; Toporowski, W.; Zielke, S. (2012): Der Handel. Grundlagen - Management - Strategien. Stuttgart.

Nakanishi, M.; Cooper, L. G. (1974): Parameter Estimation for a Multiplicative Competitive Interaction Model. Least Squares Approach. In: Journal of Marketing Research 11, 3, 303-311. doi: $10.2307 / 3151146$

Osterhage, F.; Thabe, S. (2012): Das neue Dortmund. Ansätze einer Reurbanisierung im Zuge des Strukturwandels. In: Brake, K.; Herfert, G. (Hrsg.): Reurbanisierung. Materialität und Diskurs in Deutschland. Wiesbaden, 287-303. doi: 10.1007/978-3-53194211-7

Reink, M. (2014): Perspektiven schwacher Zentren. Zusammenhänge von Handels- und Stadtentwicklung. In: Raumplanung 176, 6, 1621.
Schürmann, C. (1999): Schöne heile Einkaufswelt. Eine Methode zur Abschätzung der wohnstandörtlichen Versorgungsqualität. Dortmund. $=$ Institut für Raumplanung, Universität Dortmund, Arbeitspapiere 167.

Söfker, W. (2013): Kommentar zu BauNVO § 1 Sonstige Sondergebiete. In: Ernst, W.; Zinkahn, W.; Bielenberg, W.; Krautzberger, M. (Hrsg.): Baugesetzbuch. Kommentar. 109. Ergänzungslieferung. München.

Steinbach, J. (2000): Verfallung und Erneuerung von Stadtteilzentren - Erfahrungen aus Wien. In: Heinritz, G.; Schröder, F. (Hrsg.): Stadtteilzentren, Ladenzeilen, Ausfallstraßen. Passau, 27-58. = Geographische Handelsforschung 4.

Temmen, B. (2012): Das übergeordnete Interesse einer ausgewogenen Einzelhandelsentwicklung in Stadt und Region auf kommunaler Ebene - aus Sicht einer (Groß)Stadt. In: Kruse, S. (Hrsg.): Handbuch Einzelhandel. Bonn. 42-59.

Uttke, A. (2009): Supermärkte und Lebensmitteldiscounter. Wege der städtebaulichen Qualifizierung. Dortmund.

Vogels, P.-H.; Holl, S.; Birk, H.-J. (1998): Auswirkungen großflächiger Einzelhandelsbetriebe. Basel/Boston/Berlin. = Stadtforschung aktuell 69 .

Wieland, T. (2014): Räumliches Einkaufsverhalten und Standortpolitik im Einzelhandel unter Berücksichtigung von Agglomerationseffekten. Theoretische Erklärungsansätze, modellanalytische Zugänge und eine empirisch-ökonometrische Marktgebietsanalyse anhand eines Fallbeispiels aus dem ländlichen Raum Ostwestfalens/Südniedersachsens. Dissertation, Universität Göttingen. 